\title{
SUPERCHARGE OPERATOR OF HIDDEN SYMMETRY IN THE DIRAC EQUATION
}

\author{
Tamari T. Khachidze and Anzor A. Khelashvili

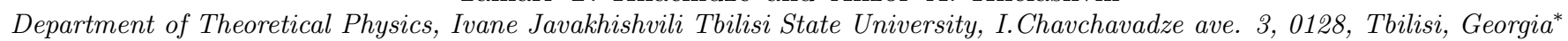

\begin{abstract}
As is known, the so-called Dirac $K$-operator commutes with the Dirac Hamiltonian for arbitrary central potential $V(r)$. Therefore the spectrum is degenerate with respect to two signs of its eigenvalues. This degeneracy may be described by some operator, which anticommutes with $K$. If this operator commutes with the Dirac Hamiltonian at the same time, then it establishes new symmetry, which is Witten's supersymmetry. We construct the general anticommuting with $K$ operator, which under the requirement of this symmetry unambiguously select the Coulomb potential. In this particular case our operator coincides with that, introduced by Johnson and Lippmann many years ago.
\end{abstract}

Key words: Dirac equation, Dirac operator, supersymmetry, commutativity, central potential, Coulomb potential, Witten's algebra.

Supersymmetry (SUSY) of the Hydrogen atom is rather old and well-studied problem. We have in mind the usual cases: SUSY in non-relativistic quantum mechanics and inclusion of spin degrees of freedom by Pauli method as well. In this last case the projection of wellknown Laplace-Runge-Lenz vector onto the electron spin direction plays the role of supercharge ${ }^{1}$.

Relatively less is known about the Dirac electron, although the so-called radial SUSY was demonstrated a long-time $\mathrm{ago}^{2}$. As for 3-dimensional case, it was shown ${ }^{3.4 .5 .6}$, that the supercharge operator is the one, introduced by Johnson and Lippmann in 1950 in the form of a brief abstract ${ }^{7}$. As regards to the more detailed derivation, to the best our knowledge, is not published in scientific literature. Moreover as far as commutativity of the Johnson-Lippmann operator with the Dirac Hamiltonian is concerned, it is usually mentioned that it can be proved by "rather tedious calculations."

The main aim of our paper is a derivation of the Johnson-Lippmann operator in a simple and transparent manner and simultaneous demonstration of its commutativity with the Dirac Hamiltonian.

Below we show that among all central potentials the Coulomb potential is a distinguished one. The additional symmetry takes place only for this potential. Then we show that the operator responsible for that symmetry reduces to the Johnson-Lippmann one in case of Coulomb potential.

So, let us consider the Dirac Hamiltonian for arbitrary central potential, $V(r)$ :

$$
H=\vec{\alpha} \cdot \vec{p}+\beta m+V(r)
$$

In this form $V(r)$ is a fourth component of a Lorentz 4 -vector. We mention this fact here because the pure Lorentz -scalar potential is also often considered $\underline{8}$.

Let us introduce the so-called Dirac operator ${ }^{9}$,

$$
K=\beta(\vec{\Sigma} \cdot \vec{l}+1)
$$

where $\vec{l}$ is the angular momentum vector, $\vec{\alpha}$ and $\beta$ are the usual Dirac matrices and $\vec{\Sigma}$ is the electron spin matrix

$$
\vec{\Sigma}=\left(\begin{array}{cc}
\vec{\sigma} & 0 \\
0 & \vec{\sigma}
\end{array}\right)
$$

It is easy to show that

$$
[K, H]=0
$$

for arbitrary central potential, $V(r)$.

Therefore the spectrum of Dirac equation is degenerate with respect to eigenvalues $\kappa$ of the $K$-operator. As a rule, this is a degeneracy with regard to the signs of $\kappa$, $( \pm \kappa)^{10}$.

We can find an operator $A$, which could connect these two signs. Naturally, such an operator should anticommute with $K$,

$$
\{A, K\}=A K+K A=0
$$

If at the same time this operator commutes with Hamiltonian $H$, it'll generate the symmetry of the Dirac equation.

Therefore, we are looking for an operator $A$ with the following properties

$$
[A, H]=0,\{K, A\}=0
$$

After that we will be able to construct supercharges as follows $3.4,5.6$

$$
Q_{1}=A, \quad Q_{2}=i \frac{A K}{|\kappa|}
$$

Then it is obvious that

$$
\left\{Q_{1}, Q_{2}\right\}=0
$$

and we can construct Witten's superalgebra, where $Q_{1}^{2}=$ $Q_{2}^{2} \equiv h$ is a Witten's Hamiltonian. Now our goal is a construction of the $A$ operator. For this purpose at first we generalize one theorem ${ }^{1.11}$, known from Pauli equation to the case of Dirac equation. For the Dirac case this theorem may be formulated as follows: 
Theorem: Suppose $\vec{V}$ be a vector with respect to the angular momentum $\vec{l}$, i.e.

$$
\left[l_{i}, V_{j}\right]=i \varepsilon_{i j k} V_{k}
$$

or, equivalently, in the vector product form one has

$$
\vec{l} \times \vec{V}+\vec{V} \times \vec{l}=2 i \vec{V}
$$

Suppose also that this vector is perpendicular to $\vec{l}$

$$
(\vec{l} \cdot \vec{V})=(\vec{V} \cdot \vec{l})=0 .
$$

Then $K$ anticommutes with operator $(\vec{\Sigma} \cdot \vec{l})$, which is scalar with respect to the total $\vec{J}$ momentum, i.e. it commutes with $\vec{J}=\vec{l}+1 / 2 \vec{\Sigma}$.

The proof of this theorem is almost trivial - it is sufficient to consider the product $(\vec{\Sigma} \cdot \vec{l})$ in this and in reversed orders and make use of definition of $K$. Then it follows that

$$
\{K, \vec{\Sigma} \cdot \vec{V}\}=K(\vec{\Sigma} \cdot \vec{V})+(\vec{\Sigma} \cdot \vec{V}) K=0
$$

It is evident that the class of operators anticommuting with $K$ (so-called, K-odd operators) is not restricted by these operators only. Any operator of the form $\hat{O}(\vec{\Sigma} \cdot \vec{l})$, where $\hat{O}$ commutes with $K$, but otherwise arbitrary, also is a $K$ - odd. Let us remark for the further application, that the following useful relation holds in the framework of conditions of the above theorem

$$
K(\vec{\Sigma} \cdot \vec{V})=-i \beta\left(\vec{\Sigma} \cdot \frac{1}{2}[\vec{V} \times \vec{l}-\vec{l} \times \vec{V}]\right)
$$

Now one can proceed to the second stage of our problem - we wish to construct the K-odd operator A, which commutes with $H$. It is clear that there remains large freedom according to the above mentioned remark about $\hat{O}$ - operator - one can take $\hat{O}$ into account or ignore it.

We have the following physically interesting vectors at hand which obey the requirements of our theorem. They are:

$$
\hat{\vec{r}} \text { - unit radius vector and } \vec{p} \text { - linear momentum vector. }
$$

Both of them are perpendicular to $\vec{l}$. Constraints of this theorem are also satisfied by Laplace-Runge-Lenz vector $\vec{A}=\hat{\vec{r}}-\frac{i}{2 m a}[\vec{p} \times \vec{l}-\vec{l} \times \vec{p}]$, but this vector is associated to the Coulomb potential. Hence, we abstain from its consideration for now. We can remark that $(\vec{\Sigma}$. $\vec{A})$ is not an independent structure. It is expressible by two other structures, e.g. $(\vec{\Sigma} \cdot \vec{A})=(\vec{\Sigma} \cdot \hat{\vec{r}})+\frac{i}{m a} \beta K(\vec{\Sigma} \cdot \vec{p})$ . Therefore, we choose the following $K$-odd terms:

$$
(\vec{\Sigma} \cdot \hat{\vec{r}}) \text { and } K(\Sigma \vec{\Sigma} \cdot \vec{p})
$$

As it turns out inclusion of $K$ into the second term of (11) is necessary for obtaining our final result. Let's remark that both operators in (11) are diagonal matrices, while the Hamiltonian (1) is nondiagonal. Therefore, in commuting of (11) with nondiagonal terms appear as well. For instance,

$$
[\vec{\Sigma} \cdot \hat{\vec{r}}, H]=\frac{2 i}{r} \beta K \gamma^{5}
$$

Therefore, we probe the following operator

$$
A=x_{1}(\vec{\Sigma} \cdot \hat{\vec{r}})+i x_{2} K(\vec{\Sigma} \cdot \vec{p})+i x_{3} K \gamma^{5} f(r)
$$

Here the coefficients $x_{i}(i=1,2,3)$ are chosen in such a way that $A$ is Hermitian operator for arbitrary real numbers and $f(r)$ is an arbitrary scalar function to be determined later.

The commutator of $A$ with $H$ is calculated straightforwardly, the result is

$$
\begin{gathered}
\quad[A, H]=x_{1} \frac{2 i}{r} \beta K \gamma^{5}+x_{2} K(\vec{\Sigma} \cdot \hat{\vec{r}}) V^{\prime}(r)- \\
-x_{3} K(\vec{\Sigma} \cdot \hat{\vec{r}}) f^{\prime}(r)-i x_{3} 2 m \beta K \gamma^{5} f(r)
\end{gathered}
$$

Equating the above expression to zero, i.e. requiring commutativity of our operator with the Dirac Hamiltonian, we find

$$
\begin{aligned}
& K(\vec{\Sigma} \cdot \hat{\vec{r}})\left[x_{2} V^{\prime}(r)-x_{3} f^{\prime}(r)\right]+ \\
& +2 i \beta K \gamma^{5}\left[\frac{x_{1}}{r}-m x_{3} f(r)\right]=0
\end{aligned}
$$

Here terms are grouped in a way that we have a diagonal matrix in the first row, while the anti-diagonal matrix is in the second row. Therefore, the two equations follow:

$$
\begin{gathered}
x_{2} V^{\prime}(r)=x_{3} f^{\prime}(r) \\
x_{3} m f(r)=\frac{x_{1}}{r}
\end{gathered}
$$

Integration of the Eq. (15) with the requirement that functions $f(r)$ and $V(r)$ tend to zero when $r \rightarrow \infty$, yields

$$
x_{3} V(r)=x_{3} f(r)
$$

while the equation (16) gives

$$
f(r)=\frac{x_{1}}{x_{2}} \frac{1}{m r}
$$

Substituting Eq. (18) into Eq (17) results in the following potential

$$
V(r)=\frac{x_{1}}{x_{2}} \frac{1}{m r}
$$

Hence, in the very general framework we have shown that the only central potential for which the Dirac Hamiltonian would have an additional symmetry (in the above mentioned sense) is a Coulomb potential. 
Meanwhile, the relative signs of coefficients $x_{1}$ and $x_{2}$ may be arbitrary. Therefore we have a symmetry both for attraction and repulsion. If we take the Coulomb potential in the usual form

$$
V_{c}(r)=-\frac{a}{r}
$$

where $a=Z e^{2}=Z \alpha$, it follows

$$
x_{2}=-\frac{1}{m a} x_{1}
$$

In this case our symmetry operator (13) becomes

$$
A=x_{1}\left\{(\vec{\Sigma} \cdot \hat{\vec{r}})-\frac{i}{m a} K(\vec{\Sigma} \cdot \vec{p})+\frac{i}{m r} K \gamma^{5}\right\}
$$

Number $x_{1}$, as an unessential common factor may be omitted. Moreover, if we make transition to the usual Dirac $\vec{\alpha}$ matrices according to the relation $\vec{\Sigma}=\gamma^{5} \vec{\alpha}$, then operator $A$ can be reduced to the form

$$
A=\gamma^{5}\left\{\vec{\alpha} \cdot \hat{\vec{r}}-\frac{i}{m a} K \gamma^{5}(H-\beta m)\right\}
$$

which coincides precisely with the Johnson-Lippmann operator $^{7}$.

We mention here that if the potential in the Dirac Hamiltonian was a Lorentz-scalar (which means the change $V \rightarrow \beta V$ ) then, while $K$ still commutes with $H$, operator $A$ does not commute anymore with $H$ even for Coulomb potential. Thus, we are convinced that in this problem of supersymmetry, the Coulomb potential (as a fourth component of 4 -vector, i.e. minimal gauge invariant switching) takes exceptional role - the supercharges and Witten algebra can be constructed only for this potential. What the real physical picture is standing behind this? Remark that taking into account the Eq. (10) for $\vec{V}=\vec{p}$, one can recast our operator for the Coulomb potential in the following form

$$
A=\vec{\Sigma} \cdot\left(\hat{\vec{r}}-\frac{i}{2 m a} \beta[\vec{p} \times \vec{l}-\vec{l} \times \vec{p}]\right)+\frac{i}{m r} K \gamma^{5}
$$

One can see that in the non-relativistic limit, i.e. $\beta \rightarrow 1$ and $\gamma^{5} \rightarrow 0$, our operator reduces to

$$
A_{N R}=\vec{\sigma} \cdot\left(\hat{\vec{r}}-\frac{i}{2 m a}[\vec{p} \times \vec{l}-\vec{l} \times \vec{p}]\right)
$$

Note the Laplace-Runge-Lenz vector in the parenthesis of Eq. (25). Therefore, relativistic supercharge reduces to the projection of the Laplace-Runge-Lenz vector on the electron spin direction. Precisely this operator, Eq.(25) was used in the case of Pauli electron ${ }^{1}$.

Therefore, we see that there is a deep relation between supersymmetry of the Dirac Hamiltonian and the symmetry related to the Laplace-Runge-Lenz vector, which appeared already in classical mechanics and provides the closeness of celestial orbits.

We can conclude that the hidden symmetry associated to the Laplace-Runge-Lenz vector governs very wide range of physical phenomena from planetery motion to fine and hyperfine structure of atomic spectra. As for the Lamb shift, which is pure quantum field theory effect, its Hamiltonian, derived by radiative corrections to a photon propagator and photon-electron vertex function, does not commute with $A$ operator and therefore spoils the above mentioned symmetry (supersymmetry). In other words, symmetry of the $A$ operator controls the absence of the Lamb shift in the Dirac theory.

In conclusion, one must also remember that the form of obtained symmetry operator is not unique. One can always replace $A \rightarrow \hat{O} A$, where $[\hat{O}, H]=0$ and $[\hat{O}, K]=0$. One can take, for example, $\hat{O}=f(K)$ - arbitrary regular matrix function of $K$. Moreover, SUSY in specific and mostly exotic models of Dirac equation (such as $2+1$ dimensions ${ }^{12}$, non-minimal or anomalous magnetic moment coupling ${ }^{13}$, squared equation ${ }^{14.15}$, etc.) are not excluded by our above consideration.
* khelash@ictsu.tsu.edu.ge

1 R. G. Tangerman, J. A. Tjon. Phys. Rev. A48,1089 (1998);

2 C. V. Sukumar, J.Phys.A: Math.Gen. 18,L697 (1985);

3 J. P. Dahl, T. Jorgensen. Int.J.Quantum Chemistry. 53,161 (1995).

4 A. A. Stahlhofen, Helv.Phys.Acta. 70, 372 (1997);

${ }^{5}$ H. Katsura, H. Aoki, ArXiv: quant-ph/0410174 (2004);

6 T. T. Khachidze, A. A. Khelashvili, Mod.Phys. Lett. A20, 2277 (2005);

7 M. H. Johnson, B. A. Lippmann, Phys.Rev. 78, 329(A), (1950);

8 C. L. Critchfield, J. Math. Phys. 17, 261 (1976);

9 P.A. Dirac, The Principles of Quantum Mechanics, 4th ed.
(Oxford Univ.Press, London, 1958). Chap.11;

10 J. J. Sakurai, Advanced Quantum Mechanics (AddisonWesley, MA,1967),Chap.3;

11 L. Biederharn, L. Louk, in Encyclopedia of Mathematics and Its Application (Addison- Wesley Reading, MA, 1981);

12 H. Ui, Progr.Theor.Phys. 72, 192 (1984); 13. V.V.Semenov. J.Phys. A: Math. Gen. 23,L721 (1990); 14. F.De Jonghe et al. Phys. Lett. B359, 114 (1995); 15. P.A.Horvathy et al. arXiv: hep-th/0006118 (2000).

13 V. V. Semenov, J.Phys. A: Math. Gen. 23,L721 (1990);

14 F. De Jonghe et al. Phys. Lett. B359, 114 (1995);

15 P. A. Horvathy et al. arXiv: hep-th/0006118 (2000). 